\title{
European Federation of Societies for Ultrasound in Medicine and Biology (EFSUMB) Position Statement on Dermatologic Ultrasound Stellungnahme der European Federation of Societies for Ultrasound in Medicine and Biology (EFSUMB) zu Dermatologischem Ultraschall
}

Authors

Fernando Alfageme ${ }^{1}$, Ximena Wortsman ${ }^{2}$, Orlando Catalano ${ }^{3}$, Gaston Roustan ${ }^{1}$, Maria Crisan $^{4}$, Diana Crisan ${ }^{5}$, Diana E. Gaitini ${ }^{6}$, Eugenio Cerezo ${ }^{7}$, Radu Badea ${ }^{8}$

Affiliations

1 Dermatology, Hospital Universitario Puerta De Hierro Majadahonda, Madrid, Spain

2 Department of Dermatology, Universidad de Chile, Santiago de Chile

3 Radiology, Instituto Pascale, Naples, Italy

4 Dermatology, University of Medicine and Pharmacy luliu Hatieganu, Cluj-Napoca, Romania

5 Dermatology, Universitätsklinikum Ulm Klinik für Dermatologie und Allergologie, Ulm, Germany

6 Radiology, Rambam Medical Center, Haifa, Israel

7 Ultrasound, Clinica DKV, Madrid, Spain

8 Regional Institute of Gastroenterology and Hepatology, University of Medicine and Pharmacy, "Iuliu Hatieganu”, Cluj-Napoca, Romania

Key words

dermatologic ultrasound, guidelines and recommendations, skin ultrasound

received 28.01.2020

accepted 09.04.2020

published online 07.05 .2020

Bibliography

Ultraschall in Med 2021; 42: 39-47

DOI 10.1055/a-1161-8872

ISSN $0172-4614$

(c) 2020. Thieme. All rights reserved.

Georg Thieme Verlag KG, Rüdigerstraße 14,

70469 Stuttgart, Germany

Correspondence

Dr. Fernando Alfageme

Dermatology, Hospital Universitario Puerta De Hierro

Majadahonda, Manuel de Falla 2, 28022 Madrid, Spain

Tel.: ++ 34/669547262

dermalfageme@gmail.com

\section{ABSTRACT}

Dermatologic ultrasound is a recent application of ultrasound for the evaluation of healthy skin and appendages and their diseases. Although the scientific literature regarding this application is still not sufficient for evidence-based guidelines, general recommendations issued by scientific societies are necessary. The EFSUMB (European Federation of Societies for Ultrasound in Medicine and Biology) steering committee for dermatologic ultrasound has developed a series of consensus position statements regarding the main fields of dermatologic ultrasound (technical requirement, normal skin and appendages, inflammatory skin diseases, tumoral skin diseases, aesthetic dermatology and practice-training requirements). This document is the foundation for future evidence-based recommendations and guidelines for dermatologic ultrasound practice.

\section{ZUSAMMENFASSUNG}

Dermatologischer Ultraschall ist eine neuere sonografische Anwendung zur Beurteilung gesunder Haut, ihrer Adnexe und deren Erkrankungen. Obwohl die wissenschaftliche Literatur zu dieser Anwendung noch nicht für evidenzbasierte Leitlinien ausreicht, sind allgemeine Empfehlungen der wissenschaftlichen Gesellschaften erforderlich. Der Lenkungsausschuss für dermatologischen Ultraschall der EFSUMB (European Federation of Societies for Ultrasound in Medicine and Biology) hat eine Reihe von Konsensus-Stellungnahmen zu den Hauptgebieten des dermatologischen Ultraschalls erarbeitet (technische Anforderungen, normale Haut und Adnexe, entzündliche Hauterkrankungen, Tumorerkrankungen der Haut, ästhetische Dermatologie und Anforderungen an die praktische Ausbildung). Dieses Dokument bildet die Grundlage für zukünftige evidenzbasierte Empfehlungen und Leitlinien für die Praxis des dermatologischen Ultraschalls. 


\section{Introduction}

Dermatologic ultrasound (DERMUS) is an application of ultrasonography (US) in the study of the normal and diseased state of the skin and appendages (nails and hair) [1].

As it is a growing, recently developed application, guidelines and recommendations based on scientific evidence are not methodologically possible. However, a position statement from a scientific society with regards to this application of US is useful for physicians involved in dermatologic US, allowing for the foundations of present clinical practice and evidence generation to be developed [2, 3].

\section{Methodological structure and classification of the consensus levels}

The executive board of the European Federation of Societies for Ultrasound in Medicine and Biology (EFSUMB) designated a dermatologic ultrasound steering committee based on qualifications including relevant publications, clinical experience and absence of conflict of interest. The Policy Document Development Strategy for Clinical Practice Guidelines, Position Statements and Technological Reviews of the EFSUMB was adhered to throughout the process for this position statement [4].

The main topics regarding dermatologic US were selected by the steering group and a comprehensive scientific literature search was performed to identify relevant studies.

Recommendations were elaborated by steering committee members and a consensus meeting for expert evaluation of these recommendations was convened at the EUROSON 2019 Congress (Granada, Spain).

A position statement was approved if $>75 \%$ of voting members were in agreement (broad agreement: $>75-95 \%$ of votes, strong consensus $>95 \%$ of the votes). For discussion a nominal group technique was applied [4]. In the case of disagreement ( $\leq 50 \%$ of the votes or less in favor) or if the rephrased or alternative position statement again failed to gain $>75 \%$ of votes, the position statement was removed. A lack of consensus on this particular issue would be recorded in the text as recommended by the EFSUMB policy document [4].

\section{Technical requirements for dermatologic ultrasound}

The main technological advance that has made dermatologic US possible is the introduction of high-frequency and very high-frequency transducers with enough spatial resolution to study the superficial structures of the skin and appendages [5, 6].

According to the DERMUS group (an international group of experts in dermatologic ultrasound), dermatologic US for the skin and appendages should be performed using a linear multiple frequency $15 \mathrm{MHz}$ transducer as the minimum standard [2].

Newer very high-frequency (> $20 \mathrm{MHz}$ ) and ultra-high-frequency transducers $(30-70 \mathrm{MHz}$ ) allow exploration of small adnexal structures, such as sebaceous glands and apocrine and eccrine glands [7].

\section{POSITION STATEMENT 1}

Operators performing a dermatology ultrasound study should be aware of patient history and clinical findings. A detailed request from the referring clinician should be available (strong agreement 9/9, 100\%).

\section{POSITION STATEMENT 2}

The minimum transducer frequency for dermatologic ultrasound should be $15 \mathrm{MHz}$. Higher transducer frequencies may provide further information that may be relevant (broad agreement 8/9, 88.9\%).

Both gel hips and gel pads, which make it possible to separate the epidermis from the transducer, should be used for accurate epidermal evaluation and avoidance of superficial vascular plexus compression [8, 9]

Also according to the DERMUS group, all US examinations should include color, power or spectral Doppler US to ascertain the presence of a vascular anomaly, providing fundamental information regarding inflammation and neovascularization $[2,10]$.

In order to detect superficial dermal, subdermal, small vessels, the pulse repetition frequency (PRF) should be adjusted accordingly and the gain should be adjusted to reduce flare artifacts [11]. Proper training in color and spectral Doppler US should be included in dermatologic US training programs [3] (see training section).

Trapezoid field-of-view (FOV) and extended FOV facilities are useful in the evaluation of large or deep lesions. Three-dimensional reconstruction software and new non-Doppler facilities for microvasculature assessment are useful and should be employed, if available on the ultrasound machine [12].

Current experience regarding dermatology application of elastography is limited, and contrast-enhanced ultrasound (CEUS) has not been deployed in dermatology US practice $[13,14]$.

\section{POSITION STATEMENT 3}

Color Doppler/power Doppler and pulsed spectral Doppler (in the case of vascular anomalies) are recommended to establish inflammatory state of skin and appendages and the presence or neovascularization (strong agreement 9/9, $100 \%$ ).

\section{Ultrasound of normal skin and appendage}

Anatomical and histological structures of skin and appendages present differential echogenicity that must be known as changes may indicate pathology $[11,15]$. 


\subsection{Ultrasound of normal skin}

Ultrasound of the skin is basically a correlation of the different layers (epidermis, dermis and subcutaneous tissue) with the acoustic interphases they produce [16].

- The epidermis is a hyperechoic line due to its keratin content,

- The dermis is a band inferior to the epidermal line which is less hyperechoic than the dermis, due to the rich collagen content

- Subcutaneous fat is a complex structure composed of hyperechoic collagen septa and hypoechoic fatty lobules

\subsection{Ultrasound of hair tracts and follicles}

A hair tract (the visible part of hair) is the final keratinized product of hair follicles, which are oblique cellular structures in the dermis $[17,18]$.

- A hair tract is a bilaminar or trilaminar lineal hyperechoic structure depending on the presence of medulla (terminal follicles) [19]

- Hair follicles are oblique hypoechoic structures that may vary in depth from subcutis to upper dermis depending on the phase of growth (early anagen follicles are deeper that superficial catagen follicles)

\subsection{Ultrasound of the normal nail apparatus}

The nail apparatus has a very close relationship with the distal interphalangeal joint.

Four elements must be assessed [20, 21]

- Nail plate: Bilaminar hyperechoic structure due to the keratin content

- Nail bed: Between nail plate and cortex of the distal phalanx

- Distal phalanx: Hyperechoic lineal interphase under the nailbed

- Nail Matrix: Ill-defined hypoechoic area that surrounds the proximal nail plate area.

\section{POSITION STATEMENT 4}

Systematic exploration of echogenicity of skin or appendages must be performed and reported as changes may indicate disease (broad agreement 8/9, 88.9\%).

\section{POSITION STATEMENT 5}

Special care should be taken in order to standardize examinations and to ensure that measurements are performed at the exact same points and with the same parameters in order to assure reproducibility of the examination (Strong agreement 9/9, 100\%).

\section{Ultrasound of skin tumors}

Ultrasound examination of the skin is widely used for the assessment of cutaneous tumors [22]. Even though histological examination remains the "gold standard" for the diagnosis of oncologi- cal skin pathology [23], US can help guide preoperative diagnosis and improve both oncological and aesthetic outcomes. Furthermore, US plays an important role not only in the therapeutic outcome after surgical management of skin tumors, but also in the follow-up process [24].

\section{POSITION STATEMENT 6}

The dermatological diagnosis of a cutaneous tumoral pathology involves clinical examination and should be confirmed by histopathology if needed (broad agreement 7/9, $77.8 \%$ ).

Modern US technology can be successfully and effectively used in the field of skin oncology, offering particular descriptive information which should be integrated in the overall examination to achieve a complex evaluation of the nature of the investigated tumor pathology. Conventional B-mode provides significant morphological data related to the tumoral lateral and depth extension, relationship to neighboring tissues (involvement of muscle, cartilage, bone), contour, echogenicity and echo-structure [14, 22]. The tumor thickness (Breslow histological index) is one of the most important prognosis factors and establishes the therapeutic strategy especially in cases of melanoma. The evidence indicates that there is a significant correlation between the sonographic "depth index" and the histological Breslow index [22, 23].

\section{POSITION STATEMENT 7}

The tumor thickness measurement should always be performed in the scan (longitudinal or transverse) that presents the maximal depth, from the point just beneath the hyperechoic band of the superficial keratin to the deepest border of the tumor invasion (broad agreement 8/9, 88.9\%).

\section{Doppler, contrast-enhanced ultrasound and elastography in skin tumors}

A color Doppler US examination combined with spectral Doppler US can reveal macro-circulation at the tumor bed level. The vascular features that may suggest a malignant lesion include hypervascularization, disorganized blood flow model with peripheral or mixed distribution, increased blood velocity and multiple vascular pedicles [23]. The lack of evidence of supply vessels in certain cases, however, reveals the limitations of Doppler US examination, which can be overcome by CEUS, which allows for assessment of the microcirculation. Analysis of the time-intensity curves during CEUS examination suggests that the vascular dynamics of the tumor depend on vascular resistance, shunts, histological type and location. The disposition of the tumor vessels, the uptake pattern of the contrast agent, the blood flow velocity are parameters that may suggest the malignant or benign nature of tumors. According to published data, malignant tumors display an inhomogeneous uptake pattern on a CEUS examination with a significantly higher value for the washout time. Compared to benign tu- 
mors, CEUS allows analysis of tumoral vascularization from the early arterial to the late venous phase, emphasizing maximal uptake, wash-up time, duration of passage and distribution pattern in the area of interest [14, 23, 24].

Strain elastography is also a useful examination in the diagnosis of skin cancer. The qualitative elastography appearance is significantly associated with semi-quantitative elastography (strain ratio) measurements. Any type of elastography provides information about tissue stiffness. According to the literature, malignant tumors display a high or medium increase in stiffness. The stiffness positively correlates with the thickness of the tumors. The role of elastography in skin tumors has not been subject to active research. As malignant tumors are stiffer than benign ones, elastography added to B-mode and color Doppler US examination has the potential to improve the accuracy of traditional clinical diagnosis $[25,26]$.

\section{POSITION STATEMENT 8}

The use of dermatologic ultrasound as an examination for palpable skin tumors is recommended, since it offers valuable information regarding tumoral extension, vascularization, delimitation and degree of compressibility for optimized treatment (broad agreement 7/9, 77.8\%).

\subsection{Ultrasonographic aspects of non-melanoma and melanoma skin cancer}

\section{Basal cell carcinoma}

Basal cell carcinoma (BCC) displays US features such as the presence of hyperechoic 'dots' or punctiform hyperechoic areas within the lesion (which correspond to calcium deposits, keratinized cells or prominent basal cell aggregates), anechoic or hyperechoic areas (suggestive for cysts, mucin deposits), the presence of two or more vascular pedicles [23]. The blood flow is more prominent at the inferior aspect of the lesion. Tumor margins are difficult to assess in cases of morpheaform and infiltrative BCC, although in most cases BCCs tend to have well-defined borders [28, 29]. The presence of cutaneous elastosis can on occasion make the exact measurement of the lesion difficult [24]. Furthermore, the hyperechoic 'dots' within the tumor, determined by the presence of keratin or basal cell nests, can be of use in differentiating a basal cell carcinoma from melanoma and even different histologic varieties of BCC $[29,30]$.

\section{Squamous cell carcinoma}

Squamous cell carcinoma (SCC) can present perpendicular shadows determined by superficial scales or crusts. Hyperkeratosis and the presence of abundant inflammatory infiltrate may lead to an overestimation of tumor size [22]. SCCs frequently invade deeper structures. Due to the risk of the development of metastasis, loco-regional lymph nodes should also be evaluated [31]. Regarding the microcirculation pattern, SCCs usually present with two or more vascular pedicles and have a mixed or peripheral intratumoral circulatory pattern [23, 24].

\section{Melanoma}

Malignant melanoma (MM) usually presents as homogeneous or inhomogeneous, hypoechoic, with an irregular contour, intense chaotic vascularization (mostly arterial vessels) [32], two or more vascular pedicles, increased echogenicity of the underlying subcutaneous tissue, increased or moderate stiffness on elastography [33]. When a melanoma is suspected, it is important to check for satellite lesions, in-transit lesions and lymph node metastasis [33, 34].

\section{Other skin malignancies}

Skin lymphoma, angiosarcoma, Kaposi sarcoma, adnexal carcinoma and other non-melanoma skin cancer (NMSC) can also be assessed using US [35-37]. Cutaneous lymphoma may appear either as a nodular or diffuse mass. The nodular mass appears solid hypoechoic and is poorly defined. The diffuse form appears as a hyperechoic, poorly defined area with increased thickness of the subepithelial layers. The thickness or degree of infiltration of cutaneous lymphoma can also be assessed by US [37]. Moreover, the changes in skin infiltration following therapy can be evaluated by B-mode US examination and elastography. An angiosarcoma can also have a characteristic malignant appearance on US examination, appearing as a hypoechoic, poorly defined lesion with intense vascularization that infiltrates deeper structures [38]. Kaposi sarcoma is seen as a hypoechoic, intensely vascularized plaque or nodule [35].

As the biology, growth rate and metastasis risk of malignant skin tumors are diverse, sonographic follow-up should be done according to the normally accepted clinical practice for skin cancer.

\section{POSITION STATEMENT 9}

Ultrasound is useful for dermatological tumor follow-up. The frequency and length of follow-up are based on each tumor type (broad agreement 8/9, 88.9\%).

\section{Inflammatory skin diseases}

Although inflammation of the skin and appendages can usually be accessed by clinical visual inspection or palpation, some deep processes are difficult to evaluate as they involve deeper cutaneous structures such as deep dermis and subcutaneous tissue [1, 8, 39]. Moreover, skin sclerosing diseases (i. e., morphea, scleroderma, chronic graft versus host disease) have episodes of inflammatory change, which are difficult to evaluate, and will require a different therapeutic approach [40]. Therefore, the role of US in these clinical scenarios is to add useful information to the clinical visual exploration for more accurate patient assessment and staging [41]. 


\subsection{General inflammatory ultrasound signs in skin diseases}

Although there is an immense variety of inflammatory diseases of the skin and appendages, common shared US findings in inflammatory skin disorders include [39]:

- Hypoechogenic areas in the subepidermal portion of the dermis

- Increased local blood flow demonstrated by color Doppler US

- Hypoechogenic septa and hyperechoic fatty lobules when the subcutaneous tissue is affected

These features help to determine the level and extent of the changes in inflammatory disorders of the skin, hair, and nails [41].

\section{POSITION STATEMENT 10}

With inflammatory diseases of the skin and appendages, the level and extent of inflammation should be assessed and reported as it may influence treatment (broad agreement $8 / 9,88.9 \%)$.

\subsection{Skin ultrasound in infectious diseases}

Ultrasound can be used to assess the extent of plantar warts and to monitor treatment response in human papillomavirus infections [42]. Ultrasound assessment of abscesses has also become widespread in emergency departments in the United States. Ultrasound evaluation in the pediatric emergency department can alter the treatment strategy (drainage vs. no drainage) in $15 \%$ of cases evaluated by physical examination alone [43]. An additional advantage of US in the diagnosis of abscesses is that it can be performed with minimal point-of-care training [44].

\section{POSITION STATEMENT 11}

Ultrasound is useful in evaluating and detecting subclinical subcutaneous abscesses in the emergency department. Ultrasound equipment and trained personnel are recommended for this kind of evaluation (broad agreement 7/9, $77.8 \%$ ).

\section{Psoriasis}

Ultrasound characteristics of psoriasis include epidermal and dermal thickening and subepidermal hypoechoic areas with increased blood flow on color Doppler US. These US findings, and in particular, dermal thickness, have been found to be correlated with disease severity measured using the Psoriasis Area Severity Index and other scales assessing the severity or extent of disease $[45,46]$. In a multicenter study by the Spanish Rheumatology Society, high-frequency US evaluation showed a reduction in plaque thickness and Doppler signal intensity in the dermis of patients treated with infliximab [47]. Psoriatic nails appear thicker and irregular mainly on the ventral aspect of the nail plate in comparison with healthy nails or nails affected by other diseases (ato- pic dermatitis, mycoses) $[48,49]$. Nail disease in psoriasis has been correlated with the presence of enthesopathy and psoriatic arthritis, even in the absence of clinical signs. Therefore, with clinically inconclusive signs of a psoriatic onychopathy, US may add useful information [50-52].

\section{POSITION STATEMENT 12}

In patients with suspected psoriatic arthritis, US findings of psoriatic onychopathy can support the diagnosis of psoriasis (strong agreement 9/9, 100\%).

\section{Hidradenitis suppurativa}

Hidradenitis suppurativa is a predominantly dermal and subcutaneous inflammation that can be assessed in detail by US. Comparing epidermal and dermal thickness in patients with hidradenitis and healthy controls, Wortsman et al. found that areas of the body affected by hidradenitis exhibited an increased dermal-epidermal thickness, areas of lower echogenicity, formed by pseudo-cysts, fistulous tracts and fluid collections that allowed US diagnosis of hidradenitis suppurativa [53, 54].

In a multicenter study ultrasound better assessed hidradenitis suppurativa patients than clinical inspection alone, resulting in upstaging of most patients as a consequence of the identification of occult and deep fistulous tracts, previously considered to be part of an inflammatory nodule [55].

\section{POSITION STATEMENT 13}

Ultrasound is recommended for supporting diagnosis, staging and treatment monitoring in hidradenitis suppurativa treatment (strong agreement 9/9, $100 \%$ ).

\section{Collagenosis}

Scleroderma is the collagenosis disease most widely studied by US, as treatment varies according to the stage of disease (inflammatory or sclerotic). In a study of 104 morphea plaques in 59 patients, Wortsman et al. [56] showed that US had a sensitivity of $100 \%$ and a specificity of $98.4 \%$ for differentiating between the inflammatory and the sclerotic phases. In the same study, US detected subclinical inflammation in five patients with ParryRomberg syndrome. Attempts have been made to standardize these results to create semi-quantitative scales to assess the effectiveness of treatments [57]. With respect to scleroderma ulcers, inflammation and superinfection can also be assessed by US as increased vascularization is evidenced on color Doppler US [58]. Calcinosis associated with scleroderma and dermatomyositis can also be detected and evaluated by US [58, 59]. Recently shear wave elastography evaluation of scleroderma patients has been confirmed to be useful for the evaluation of generalized scleroderma patients [60]. 


\section{POSITION STATEMENT 14}

Ultrasound is recommended for supporting diagnosis, activity assessment and follow-up in scleroderma/morphea patients (broad agreement 8/9, 88.9\%).

\section{Aesthetic dermatologic ultrasound}

The number of aesthetic procedures has been increasing explosively worldwide over the last decade, and most of these techniques are performed blindly, sometimes in different institutions and by operators with variable levels of training from medical and non-medical backgrounds. Moreover, patients may forget or be unaware of the type of treatment that they received [61, 62]. Thus, anatomical information can be challenging to obtain, but is critical for management and outcome in aesthetic medicine, where good results are the goal, and scars and complications are unwanted. The use of US is expanding in aesthetic medicine since US can provide relevant information that includes data on facial anatomical variants, the type, location and extent of common cosmetic fillers, the identification of implants, the complications of lipolytic procedures, and the possibility of percutaneous US guidance for the procedure $[62,63]$.

\subsection{Main indications for ultrasound in aesthetic medicine}

\section{1 - Assessment of photoaging}

Ultrasound can detect and measure signs of photoaging caused by prolonged exposure to the sun through the observation of the subepidermal low echogenic band (SLEB) which is produced by the deposit of glycosaminoglycans in the papillary dermis (upper dermis) [64].

\section{POSITION STATEMENT 15}

Ultrasound is recommended as a valuable tool to detect and quantify photoaging (broad agreement 8/9, 88.9\%).

\section{2- Recognition of relevant anatomical data}

Anatomical variants in vessels, muscles or glands, measurement of the thickness of skin layers and the assessment of blood flow in complications are relevant for planning or managing cosmetic techniques and complications. Moreover, the face, the most common corporal region for cosmetic procedures, presents a complex anatomical structure where the skin layers are thinner in comparison to other body regions, and any abnormality is highly visible $[65,66]$.

\section{POSITION STATEMENT 16}

Ultrasound is recommended as a valuable tool for evaluating facial anatomical structures in subjects before and after aesthetic procedures (broad agreement 7/9, 77.8\%).

\section{3- Management of cosmetic fillers}

Ultrasound allows for the detection and identification of common cosmetic fillers as well as the assessment of their location and extent and potential complications. These fillers include deposits such as hyaluronic acid, polymethylmethacrylate, silicone (pure or oily forms), calcium hydroxyapatite, polyacrylamide, and polycaprolactone that are approved by the Food and Drug Administration (FDA) and European Union Medical Agencies as well as those that are not approved. Percutaneous US guidance of the injection of hyaluronidase has also been reported in the management of hyaluronic acid complications [67-69].

\section{4- Detecting implants and their complications}

Ultrasound can support the detection of organic and synthetic implants and their complications. Examples of organic implants are fat, cartilage and bone grafts. Examples of synthetic implants are pure silicone, polyethylene, and polydioxanone (e.g. tensor threads). Complications of implants include an excessively superficial location, extrusion, chronic inflammatory and fibrotic reactions as well as rupture $[62,65,69-71]$.

\section{POSITION STATEMENT 17}

Ultrasound is recommended as a valuable tool to detect and identify common types of cosmetic fillers and organic or synthetic implants and is recommended in the management of their complications (strong agreement 9/9, $100 \%$ ).

\section{5- Detection of complications of lipolytic procedures}

The goal of these procedures is to decrease the amount of hypodermal fatty tissue using techniques that generate inflammation and liquefaction of the fat. These procedures include radiofrequency, mesotherapy or cryolipolysis. Ultrasound can provide anatomical information for planning these techniques, detect the extent and location of the inflammatory changes and may serve as a monitoring technique for the assessment of the outcomes or the management of potential complications [62, 72].

\section{POSITION STATEMENT 18}

Ultrasound is a valuable tool to support planning and detection of anatomical changes, assessment of outcomes and management of complications of lipolytic procedures (strong agreement 9/9, $100 \%$ ). 


\section{6- Detection of complications of surgical aesthetic procedures}

Ultrasound can detect complications of cosmetic or plastic surgery procedures, such as dermal and hypodermal edema, lymphedema, seromas, hematomas, abscesses, fistulous tracts, fat necrosis, granulomas and loose sutures [62, 65].

\section{POSITION STATEMENT 19}

Ultrasound is recommended for the evaluation of plastic surgery procedures and their complications (broad agreement $7 / 9$, $77.8 \%)$.

\section{7- Percutaneous ultrasound guidance of non-surgical and surgical aesthetic procedures}

Percutaneous ultrasound guidance of aesthetic or plastic surgery procedures can improve the precision of injections, drainage procedures and corrections and decrease potential adverse reactions or complications due to injury to neighboring structures [73, 74].

\section{POSITION STATEMENT 20}

Percutaneous ultrasound guidance of non-surgical and surgical aesthetic procedures is recommended as it increases precision and decreases the potential for unwanted effects or neighboring injuries (strong agreement 9/9, $100 \%$ ).

\section{Professional requirements, reports and training and accreditation in dermatologic ultrasound}

\subsection{Standard professional practical issues}

Operators should be trained in US imaging and have knowledge of dermatologic pathologies to be able to better perform and interpret examinations [1-3]. Regarding the certification required to perform dermatologic US, a physician (not a sonographer) should be the person in charge of performing examinations. The main reason for this statement is that the clinical dermatologic diagnosis must be determined by the operator and that is not within the scope of non-physician operators [2]. Regarding the number of examinations performed during training and competence maintenance, the DERMUS guidelines, based on expert opinion, suggest a minimum of 30 supervised examinations for assessing competence and a minimum of 300 examinations per year for competence maintenance [3].

\section{POSITION STATEMENT 21}

Dermatological US should be performed by an adequately trained physician with knowledge of both clinical and sonographic dermatology (strong agreement 9/9, $100 \%$ ).

\subsection{Dermatologic ultrasound examination reporting}

A written report of the findings has to be produced following dermatological US. A standardized report of the US examination is recommended and images should be kept together with the report, ideally on a computer-based picture archiving system (PACS) and electronic patient notes (EPR). Documentation of the exact anatomical position of US findings, for example using anatomical position markers or text within the images describing the location of the finding, is also recommended. Dermatologic US examinations should be correlated with the clinical and histologic results $[2,75]$. Coordinated interdisciplinary collaboration among the physicians involved in dermatological diagnosis and treatment, i. e., radiologist, dermatologist, plastic surgeon, and pathologist, is desirable.

\section{POSITION STATEMENT 22}

A written report must be provided after a dermatologic US examination. High quality images should be recorded, stored and made available for follow-up examinations (broad agreement $8 / 9,88.9 \%)$

\subsection{Dermatologic ultrasound education}

Theoretical and practical educational courses are needed to improve the performance and interpretation of dermatological US [3]. Two different course models should be organized to provide a minimum of two levels of complexity: basic and advanced. Educational course length should be a minimum of two days: theory (one day) and practical training (one day). One experienced instructor per five participants is recommended for the practical part of the course $[3,76,77]$. Educational courses should include US-guided interventions (biopsies, injections, ablations) of dermatological lesions. Courses should be accredited by EFSUMB or any other national ultrasound society that is part of EFSUMB or a dermatological scientific society.

\section{POSITION STATEMENT 23}

Basic courses on dermatologic US should be both theoretical and practical with a minimum of two days of training. Clinical images should be available for training since clinical-sonographic correlation is key in this application (broad agreement $8 / 9,88.9 \%)$. 


\section{POSITION STATEMENT 24}

Ongoing practice is encouraged in order to maintain skills and competence. Ultrasound information should be integrated with clinical and histopathological data (strong agreement 9/9, $100 \%)$.

\section{Conflict of Interest}

The authors declare that they have no conflict of interest.

\section{Acknowledgements}

We kindly acknowledge the continuous help and motivation of Mrs. Lynne Rudd and the experienced and the wise help in methodology to Prof. P. Sidhu and Dr. C. Jenssen.

\section{References}

[1] Wortsman X. Common Applications of Dermatologic Sonography. Journal of Ultrasound in Medicine 2012; 31: 97-111

[2] Wortsman X, Alfageme F, Roustan G et al. Guidelines for Performing Dermatologic Ultrasound Examinations by the DERMUS Group. J Ultrasound Med 2016; 35: 577-580

[3] Wortsman X, Alfageme F, Roustan G et al. Proposal for an Assessment Training Program in Dermatologic Ultrasound by the DERMUS Group. J Ultrasound Med 2016; 35: 2305-2309

[4] Jenssen C, Gilja OH, Serra AL et al. European Federation of Societies for Ultrasound in Medicine and Biology (EFSUMB) Policy Document Development Strategy - Clinical Practice Guidelines, Position Statements and Technological Reviews. Ultrasound Int Open 2019; 5: E2-E10

[5] Kleinerman R, Whang TB, Bard RL et al. Ultrasound in dermatology: principles and applications. J Am Acad Dermatol 2012; 67: 478-487

[6] Ulrich J, Schwürzer-Voit M, Jenderka K et al. Sonographic diagnostics in dermatology. JDDG: Journal der Deutschen Dermatologischen Gesellschaft 2014; 12: 1083-1099

[7] Wortsman X, Carreño L, Ferreira-Wortsman C et al. Ultrasound Characteristics of the Hair Follicles and Tracts, Sebaceous Glands, Montgomery Glands, Apocrine Glands, and Arrector Pili Muscles. J Ultrasound Med 2019; 38: 1995-2004

[8] Wortsman $X$ et al. Clinical usefulness of variable-frequency ultrasound in localized lesions of the skin. Journal of the American Academy of Dermatology 2010; 62: 247-256

[9] Echeverría-García B, Borbujo J, Alfageme F. The use of ultrasound imaging in dermatology. Actas Dermosifiliogr 2014; 105: 887-890

[10] Alfageme F. Broadening the Sonographic Spectrum of Vascular Anomalies. Actas Dermosifiliogr 2018; 109: 199

[11] Alfageme Roldán F, Mollet Sánchez J, Cerezo López E. Physical principles and general considerations. Actas Dermosifiliogr 2015; 106: 3-9

[12] Salgüero Fernández I, Alfageme Roldán F, Roustan Gullón G. Greater detection of small caliber blood vessels in port wine xtain with $X$ mode than with conventional Doppler: A Pilot Study. Actas Dermosifiliogr 2017; 108: 690-692

[13] Alfageme F. Elastography in dermatology. Actas Dermosifiliogr 2016; 107: $652-660$
[14] Crisan D, Crisan M, Badea R et al. Integrative analysis of cutaneous skin tumours using ultrasonogaphic criteria. Preliminary results. Medical Ultrasonography 2014; 16: 285-290

[15] Welzel J, Schuh S. Noninvasive diagnosis in dermatology. Journal der Deutschen Dermatologischen Gesellschaft 2017; 15: 999-1016

[16] de Troya Martín M, Alfageme Roldán F. Ultrasound in dermatology: a new approximation to knowledge of the skin. Actas Dermosifiliogr 2015; 106: $1-2$

[17] Wortsman X, Wortsman J, Matsuoka L et al. Sonography in pathologies of scalp and hair. Br J Radiol 2012; 85: 647-655

[18] Wortsman X, Roustan G, Martorell A. Color Doppler ultrasound of the scalp and hair. Actas Dermosifiliogr 2015; 106: 67-75

[19] Wortsman X, Guerrero R, Wortsman J. Hair morphology in androgenetic alopecia: sonographic and electron microscopic studies. J Ultrasound Med 2014; 33: 1265-1272

[20] Wortsman X, Jemec GB. Ultrasound imaging of nails. Dermatol Clin 2006; 24: 323-328

[21] Vidal D, Echeverria B, García-Gavín J et al. Ultrasound in the management of nail disease. Actas Dermosifiliogr 2015; 106: 60-66

[22] Crisan M, Crisan D, Sannino G et al. Ultrasonographic staging of cutaneous malignant tumors: an ultrasonographic depth index. Arch Dermatol Res 2013; 305: 305-313

[23] Crisan D, Crisan M, Badea R et al. Integrative analysis of cutaneous skin tumours using ultrasonogaphic criteria. Preliminary results. Medical Ultrasonography 2014; 16: 285-290

[24] Barcaui Ede O, Carvalho AC, Lopes FP et al. High frequency ultrasound with color Doppler in dermatology. An Bras Dermatol 2016; 91: 262273

[25] Increased Marginal Stiffness Differentiates Infiltrative From Noninfiltrative Cutaneous Basal Cell Carcinomas in the Facial Area: A Prospective Study. J Ultrasound Med 2019; 38: 1841-1845

[26] Dasgeb B, Morris MA, Mehregan D et al. Quantified ultrasound elastography in the assessment of cutaneous carcinoma. $\mathrm{Br}$ J Radiol 2015; 88: 20150344

[27] MacFarlane D, Shah K, Wysong A et al. The role of imaging in the management of patients with nonmelanoma skin cancer: Diagnostic modalities and applications. J Am Acad Dermatol 2017; 76: 579-588

[28] Wortsman X. Sonography of facial cutaneous basal cell carcinoma: a first-line imaging technique. J Ultrasound Med 2013; 32: 567-572

[29] Vega N, Wortsman X, Navarrete N et al. Color Doppler Ultrasound Supports Early Diagnosis of Mixed High and Low Risk of Recurrence Subtypes in the Same Basal Cell Carcinoma Lesion. Dermatol Surg 2018; 44: 741-743

[30] Wortsman X, Vergara P, Castro A et al. Ultrasound as predictor of histologic subtypes linked to recurrence in basal cell carcinoma of the skin. J Eur Acad Dermatol Venereol 2015; 29: 702-707

[31] Que SKT, Zwald FO, Schmults CD. Cutaneous squamous cell carcinoma: Incidence, risk factors, diagnosis, and staging. J Am Acad Dermatol 2018; 78: 237-247

[32] Catalano O, Setola SV, Vallone P et al. Sonography for locoregional staging and follow-up of cutaneous melanoma: how we do it. J Ultrasound Med 2010; 29: 791-802

[33] Catalano O, Caracò C, Mozzillo N et al. Locoregional spread of cutaneous melanoma: sonography findings. Am J Roentgenol 2010; 194: 735-745

[34] Catalano O, Siani A. Cutaneous melanoma: role of ultrasound in the assessment of locoregional spread. Curr Probl Diagn Radiol 2010; 39: 30-36

[35] Carrascosa R, Alfageme F, Roustán G et al. Skin ultrasound in Kaposi sarcoma. Actas Dermosifiliogr 2016; 107: e19-22

[36] Catalano O, Alfageme Roldán F, Scotto di Santolo M et al. Color Doppler Sonography of Merkel Cell Carcinoma. J Ultrasound Med 2018; 37: 285292 
[37] Mandava A, Koppula V, Wortsman X et al. The clinical value of imaging in primary cutaneous lymphomas: Role of high resolution ultrasound and PET-CT. Br J Radiol 2019; 92: 20180904

[38] Tomich J, Grove Nigro K, Barr RG. Primary Angiosarcoma of the Breast: A Case Report and Review of the Literature. Ultrasound Q 2017; 33: 46-48

[39] Wortsman X, Jemec G. Common inflammatory diseases of the skin: From the skin to the screen. Adv Psoriasis Inflammatory Skin Dis 2010; 2: 9-15

[40] Wortsman X, Wortsman J, Sazunic I et al. Activity assesment in morphea using color doppler ultrasound. J Am Acad Dermatol 2011; 65: 942-948

[41] Alfageme Roldan F. Ultrasound skin imaging. Actas Dermosifiliogr 2014; 105: 891-899

[42] Wortsman X, Jemec GB, Sazunic I. Anatomical detection of inflammatory changes associated with plantar warts by ultrasound. Dermatology 2010; 220: 213-217

[43] Ramirez-Schrempp D, Dorfman DH, Baker WE et al. Ultrasound softtissue applications in the pediatric emergency department: to drain or not to drain? Pediatr Emerg Care 2009; 25: 44-48

[44] Squire BT, Fox JC, Anderson C. ABSCESS: applied bedside sonography for convenient evaluation of superficial soft tissue infections. Acad Emerg Med 2005; 12: 601-606

[45] Gutierrez M, Wortsman X, Filippucci E et al. High-frequency sonography in the evaluation of psoriasis: nail and skin involvement. J Ultrasound Med 2009; 28: 1569-1574

[46] Gutierrez M, Filippucci E, Bertolazzi C et al. Sonographic monitoring of psoriatic plaque. J Rheumatol 2009; 36: 850-851

[47] De Agustín JJ, Moragues C, De Miguel E et al. A multicentre study on high-frequency ultrasound evaluation of the skin and joints in patients with psoriatic arthritis treated with infliximab. Clin Exp Rheumatol 2012; 30: $879-885$

[48] Gutierrez M, Wortsman X, Filippucci E et al. High-frequency sonography in the evaluation of psoriasis: nail and skin involvement. J Ultrasound Med 2009; 28: 1569-1574

[49] Moreno M, Lisbona MP, Gallardo F et al. Ultrasound Assessment of Psoriatic Onychopathy: A Cross-sectional Study Comparing Psoriatic Onychopathy with Onychomycosis. Acta Derm Venereol 2019; 99: 164-169

[50] Krajewska-Włodarczyk M, Owczarczyk-Saczonek A, Placek W et al. Distal interphalangeal joint extensor tendon enthesopathy in patients with nail psoriasis. Sci Rep 2019 Mar 2019; 9: 3628

[51] Vidal D, Alfageme F, Ruiz-Villaverde R et al. Ultrasound Characterization of Psoriasis of the Nails: A Case-Control Study. Actas Dermosifiliogr 2017; 108: 968-996

[52] Naredo E, Janta I, Baniandrés-Rodríguez O et al. To what extend is nail ultrasound discriminative between psoriasis, psoriatic arthritis and healthy subjects? Rheumatol Int 2019; 39: 697-705

[53] Wortsman X, Jemec GBE. High frequency ultrasound for the assessment of hidradenitis suppurativa. Dermatol Surg 2007; 33: 1-3

[54] Wortsman X, Moreno C, Soto R et al. Ultrasound in-depth characterization and staging of hidradenitis suppurativa. Dermatol Surg 2013; 39: 1835-1842

[55] Martorell A, Alfageme Roldán F, Vilarrasa Rull E et al. Ultrasound as a diagnostic and management tool in hidradenitis suppurativa patients: a multicentre study. J Eur Acad Dermatol Venereol 2019; 33: 21372142. doi:10.1111/jdv.15710 [Epub ahead of print]

[56] Wortsman X, Wortsman J, Sazunic I et al. Activity assessment in morphea using color Doppler ultrasound. J Am Acad Dermatol 2011; 65: 942-948

[57] Li SC, Liebling MS, Haines KA et al. Initial evaluation of an ultrasound measure for assessing the activity of skin lesions in juvenile localized scleroderma. Arthritis Care Res 2011; 63: 735-742
[58] Suliman YA, Kafaja S, Fitzgerald J et al. Ultrasound characterization of cutaneous ulcers in systemic sclerosis. Clin Rheumatol 2018; 37: 15551561

[59] Lorente M, Alfageme F, González C. Ultrasound Diagnosis of Calcified Skin Deposits. Actas Dermosifiliogr 2015; 106: 586-588

[60] Wang Y, Shan JL, Chen HY et al. Comparison of 2-D shear wave elastography with clinical score in localized scleroderma: A new method to increase the diagnostic accuracy. Dermatol 2019; 46: 131-138

[61] Wortsman X. Sonography of cosmetic procedures. In: Wortsman X, Jemec GBE, eds.; Dermatologic Ultrasound with Clinical and Histologic Correlations. 1st ed New York, NY: Springer NY; 2013: 373-399

[62] Wortsman X. Common Applications of Ultrasound in Cosmetics and Plastic Surgery. In: Wortsman X, ed.; Atlas of Dermatologic Ultrasound. 1st ed New York: Springer; 2018: 179-214

[63] Schelke LW, Van Den Elzen H], Erkamp PPM et al. Use of ultrasound to provide overall information on facial fillers and surrounding tissue. Dermatologic Surg 2010; 36: 1843-1851

[64] Gniadecka M, Gniadecki R, Serup J et al. Ultrasound structure and digita image analysis of the subepidermal low echogenic band in aged human skin: Diurnal changes and interindividual variability. J Invest Dermatol 1994; 102: 362-365

[65] Wortsman X, Wortsman J. Sonographic outcomes of cosmetic procedures. Am J Roentgenol 2011; 197: W910-W918

[66] Kwon HJ, Kim B], Ko EJCS. The Utility of Color Doppler Ultrasound to Explore Vascular Complications After Filler Injection. Dermatol Surg 2017; 43: 1508-1510

[67] Wortsman X. Identification and complications of cosmetic fillers: Sonography first. J Ultrasound Med 2015; 34: 1163-1172

[68] U.S. Food and Drug Administration. Dermal Fillers. https://www.fda. gov/MedicalDevices/ProductsandMedicalProcedures/CosmeticDevices/ ucm619837.htm Published 2018. Accessed 2018 September 14

[69] Villegas Fernández C, Burón CÁlvarez I, Fernández-Tresguerres Centeno A et al. Ecografía cutánea y rellenos dermatológicos. Actas Dermosifiliogr 2015; 106: 87-95

[70] Grippaudo FR, Mattei M. High-frequency sonography of temporary and permanent dermal fillers. Ski Res Technol 2010; 6: 265-269

[71] Pérez-Pérez L, García-Gavín J, Wortsman X et al. Delayed adverse subcutaneous reaction to a new family of hyaluronic acid dermal fillers with clinical, ultrasound, and histologic correlation. Dermatologic Surg 2017: 43: 605-608

[72] Cassuto D, Pignatti M, Pacchioni L et al. Management of complications caused by permanent fillers in the face: A treatment algorithm. Plast Reconstr Surg 2016; 138: 215e-227e

[73] Quezada-Gaón N, Wortsman X. Ultrasound-guided hyaluronidase injection in cosmetic complications. J Eur Acad Dermatology Venereol 2016; 30: e39-e40

[74] Quezada-Gaon N, Wortsman X, Peñaloza O et al. Comparison of clinical marking and ultrasound-guided injection of Botulinum type $\mathrm{A}$ toxin into the masseter muscles for treating bruxism and its cosmetic effects. J Cosmet Dermatol 2016; 5: 238-244

[75] Martorell A, Wortsman X, Alfageme F et al. Ultrasound Evaluation as a Complementary Test in Hidradenitis Suppurativa: Proposal of a Standarized Report. Dermatol Surg 2017; 43: 1065-1107

[76] Alfageme F, Cerezo E, Fernandez IS et al. Introduction of basic dermatologic ultrasound in undergraduate medical education. Ultrasound Int Open 2016; 2: E136-E139

[77] Spanish Society of Ultrasound. Accreditation in Dermatology and aesthetic medicine. http://www.seeco.es/index.php/en/sociedadespanola-de-ecografia-2/acreditacion-2 Accessed 2019 August 30 $0^{\text {th }}$ 